\section{P171 (continued)}

Objective: The project aimed to reduce the prevalence of underweight among young children and improve the nutrition knowledge of mothers and caregivers.

Study Design, Setting, Participants, Intervention: A quasi-experimental design was used in this study among young children and their mothers or caregivers in selected villages in the Philippines with high prevalence of malnutrition among children aged $0-5$ years old. The intervention group children were fed daily for 120 days with rice-mongo based complementary foods rich in energy and protein while their mothers/caregivers participated in nutrition education sessions. The non-intervention group received the regular services in the locality.

Outcome Measures and Analysis: Anthropometric measurements were taken using calibrated weighing scale. Faceto-face interview using pre-tested questionnaires was utilized to obtain the profile of program participants and knowledge of mothers/caregivers on health and nutrition. Energy and protein intakes of children using 24-hour food recall were recorded. Data were collected before and after the intervention.

Results: The prevalence of underweight children decreased significantly in the intervention compared to the non-intervention group. Energy and protein intakes among children, as well as the mean scores on nutrition knowledge among mothers/caregivers increased significantly at endline in the intervention group. The complementary feeding component of the intervention proved effective in improving the nutritional status of children and the nutrition education component increased mean scores of mothers/caregivers on health and nutrition knowledge.

Conclusions and Implications: The combined intervention of complementary feeding of children and nutrition education of mothers provided policy directions for local government units to adopt the intervention as a component of the local health and nutrition services.

Funding: Department of Science and Technology_GIA.

\section{P172 Food Appeal and Taste Perceptions Differ by School Lunch Participation During a Chef-Based Intervention}

An Nguyen, BS, Oklahoma State University;

Deana Hildebrand, PhD, LD, RDN, SNS,

deana.hildebrand@okstate.edu, Oklahoma State

University, 301 Human Sciences, Stillwater, OK 74078;

Gail Gates, PhD, RDN, FAND; Barbara Brown, PhD, RDN

Background (Background, Rationale, Prior Research, and/or Theory): Chef-based training programs addressing culinary skills of school nutrition professionals are a growing trend. Studies of these interventions reflect better compliance with the 2012 USDA school meal regulations; less is known if they influence satisfaction of school meals among students, parents and faculty.

Objective: Investigate if a chef-based intervention, Cooking for Kids, influenced stakeholder groups' satisfaction with the lunch program. The study was conducted in 17 school districts that participated in the 9-month program. The pre/ post survey was based on the satisfaction scale developed by the Child Nutrition Institute, and included a question assessing frequency of participation and allowed for open-ended comments.

Outcome Measures and Analysis: Scale items and overall satisfaction were compared using a 2x3 analysis of variance for time and frequency of participation. Comments were coded by theme; frequency analysis was used to identify common themes. Chi-square was used to determine if the proportion of negative versus positive survey comments changed over time.

Results: The number of stakeholders that completed the survey were 3,820 students, 660 parents, and 364 faculty. For each stakeholder group, regardless of participation frequency, there were no changes in overall satisfaction from pre- to post- intervention. Across all groups, stakeholders who always participated reported higher overall satisfaction compared to those who sometimes or never participated $(P<.001)$. Faculty had a significant shift in the proportion of negative to positive comments $(P=.004)$. The prevalent themes were related to taste and appeal of the food.

Conclusions and Implications: Stakeholders who participate in the school lunch program are likely to be satisfied with the meals, compared to those who never participate. Appeal and taste tend to drive satisfaction. Soliciting input for school menus and introducing new foods with taste testings, among both participants and nonparticipants, may help increase stakeholders' satisfaction. Funding: Oklahoma State Department of Education, USDA.

\section{P173 How Students View Food Security: Results From Cognitive Interviews With Undergraduates at a Land-Grant University}

Cassandra J. Nikolaus, MS, University of Illinois, Urbana-Champaign; Brenna Ellison, PhD;

Sharon M. Nickols-Richardson, PhD, RD, nickrich@illinois.edu, University of Illinois, UrbanaChampaign, 905 S Goodwin Avenue, Urbana, IL 61801

Background (Background, Rationale, Prior Research, and/or Theory): In the U.S., food insecurity (FI) affects one in eight households, putting individuals at greater risks for compromised mental and physical wellbeing. Recent evidence suggests that post-secondary (i.e. college) students are at an increased risk of FI. However, little is known about how students interpret established FI questionnaire items.

Objective: The current study aims to understand how students think about FI questionnaire items and compare their response choices to their experiences.

Study Design, Setting, Participants, Intervention: Thirty-three undergraduate students, aged 18- to 24years old and fluent in English were recruited. Students completed the 10-item USDA Adult Food Security Survey Module (AFSSM) in-person, and then were cognitively interviewed to understand how they

Continued on page 590 